\title{
PRECATACLYSMIC BINARIES IN THE CENTRE OF
}

\section{PLANETARY NEBULAE}

\author{
G. JASNIEWICZ and A. ACKER \\ URA 1280 \\ Observatoire de Strasbourg \\ 11 , rue de l'Université \\ 67000 STRASBOURG, France
}

\begin{abstract}
We report at first on two old and large planetary nebulae (PN) of which central stars could satisfy the Ritter's criteria (1986) for being precataclysmic binaries: LoTr5 and Abell 35. Both nebulae have probably been ejected as a consequence of common-envelope evolution. A model of cataclysmic binary (CB) for the central star of Abell 35 has been tentatively attempted by Acker and Jasniewicz (1990). The nucleus of LoTr5 is a triple star (Jasniewicz et al., 1987; Malasan et al., 1991): action of a third body on the separation of the close binary could make this binary evolve into a CB (see Mazeh and Shaham, 1979). We report at second on the similarity between the spectrum of the central star of the extended PN HFG1 with that of a CB (Acker and Stenholm, 1990). The PN cited above could be fundamental objects just at the transition between the stage $P N$ and the stage $C B$.
\end{abstract}

Abell 35 and LoTr5 are very old extended PN with a visible central star of late spectral type. The IUE satellite has revealed an extremely hot companion, discovered for Abell 35 by Grewing and Bianchi (1989), for LoTr5 by Feibelman and Kahler (1983). The central stars of LoTr5 and Abell 35 could be pre-CBs; according to Ritter (1986) a detached binary can be classified as pre-CB if it satisfies the properties: the primary component is a white dwarf or a white dwarf precursor; the secondary component is an essentially unevolved star of low mass $\left(M_{2} \leq 1 M_{\odot}\right)$; the orbital period is so short $\left(P \leq 2^{d}\right)$ that the system can only have been formed via a common-envelope evolution; the binary is the central star of a PN.

The central star of the PN HFG1 presents some characteristics of an extreme member of the long-period intermediate polar class of magnetic CBs. Besides, two CBs, $063+71$ and GK per, are surrounded by nebulae resembling old PNs (Bond, 1989). More, Krautter and Radons (1986) also wonder whether the CB $623+71$ is the central star of a PN.

\section{References}

Acker, A., Jasniewicz, G.: 1990, $A \mathscr{E} A$ 238, 325

Acker, A., Stenholm, B.: 1990, $A \& A$ 233, L1

Bond, H.E.: 1989, in IAU Symp. No. 131, Planetary Nebulae, ed. Torres-Peimbert (Dordrecht:Reidel) , p.251

Feibelman, W.A., Kahler, J.B.: 1983, ApJ 269, 592

Grewing, M., Bianchi, L.: 1989, in IAU Symp. No. 131, Planetary Nebulae, ed. Torres-Pesmbert (Dordrecht:Reidel) , p.314

Jasniewicz, G., Duquennoy, A., Acker, A.: 1987, $A \& A$ 180, 145

Krautter, J., Radons, G.: 1986, Mitt. Astron. Ges. 67, 308

Malasan, H.L., Yamasaki, A., Kondo, M.: 1991, AJ 101, 2131

Mazeh, T., Shaham, J.: 1979, $A \& A$ 77, 145

Ritter, H.: 1986, $A \& A 169,139$ 